Sara Galletti, Le palais du Luxembourg de Marie de Médicis, 1611-1631

Paris, Picard, coll. « De Architectura », 2012, 294 p., 53 euros.

\title{
Thierry Verdier
}

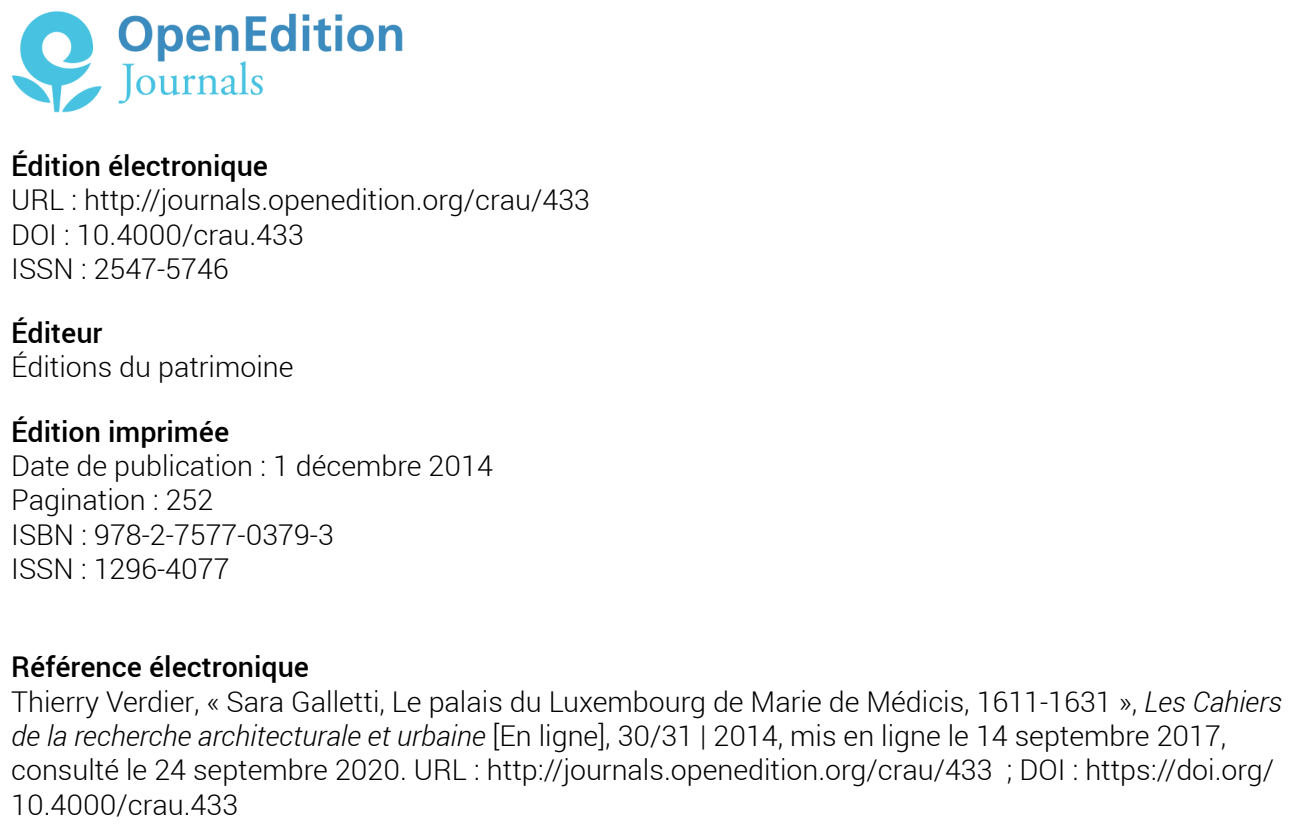




\section{Sara Galletti, Le palais du Luxembourg de Marie de Médicis, 1611-1631}

Paris, Picard, coll. "De Architectura », 2012, 294 p., 53 euros.

L'architecture du palais du Luxembourg a toujours été considérée comme une importation française des modèles palatiaux florentins du Quattrocento. Le jeu de bossage de ses façades, les volumes puissants de ses corps d'habitation, les modénatures rigoureuses du grand frontispice etc., ont souvent présenté ce dado comme une sorte d'unicum dans I'histoire de I'architecture française de l'époque classique. La formule de "Pitti parisien" qui se retrouve fréquemment sous la plume des auteurs, a longtemps suffit pour désigner ce vaste palais construit par Marie de Médicis sur la rive droite de la Seine. Depuis Louis Hautecœur ${ }^{1}$, Rosalys Coope ${ }^{2}$ ou même Anthony Blunt ${ }^{3}$, le palais parisien est présenté comme la transposition de la demeure florentine dans laquelle la veuve d'Henri IV avait grandi, et dans laquelle elle avait vécu en orpheline, trop tôt privée de ses parents ${ }^{4}$. Les historiens romantiques et feuilletonistes du XIX siècle, insistant sur le tragique d'une enfance marquée par l'assassinat de son père, François ler de Médicis et la disparition de sa mère Jeanne d'Autriche, ont voulu voir en cette reine, la figure triste d'une grande princesse

1. Louis Hautecœur, Histoire de I'Architecture classique en France. T. 1 III, L'architecture sous Henri IV et Louis XIII, Paris, éditions A. et J. Picard, 1966-1967.

2. Rosalys Coope, Salomon de Brosse and the Development of the Classical Style in French Architecture, Londres, A. Zwemmer Ltd, 1972, p. 110-134.

3. Anthony Blunt, Art and architecture in France 1500-1700. Londres, The Pelican exilée. Élevée dans le luxe et les arts, dans le bel esprit du clan Médicis, dans la quête curieuse des hommes de sciences, mais aussi dans l'irrésolution chronique des intrigants et des ambitieux, la piccola duchessa voyait le monde comme une vaste cour dans laquelle Castiglione aurait présidé en Phénix.

Dès lors, beaucoup ont voulu voir dans son mariage avec le volage et brutal roi de France un déchirement, une sorte de rupture brutale avec les lieux et les amitiés qui lui avaient permis de supporter sa condition d'orpheline isolée dans un monde d'adultes. Pour tous, il semblait logique, pratiquement évident, qu'elle n'eût de cesse, une fois expatriée dans les terres froides de l'île-de-France, au sein d'une cour bien peu courtoise, de retrouver une part de "sa Florence ». Le palais du Luxembourg en devenant en quelque sorte, le témoignage.

Celle que les médisants nommaient "la grande banquière " - par allusion à la formidable dot de 600000 écus d'or qu'elle glissa dans sa corbeille de mariée - n'était alors qu'une héritière. Sa fortune tombait à point nommé. II n'aurait été question de faire de cette femme autre chose que ce qu'exigeait l'étiquette et si elle se frappait d'architecture au point $d^{\prime}$ 'importer à Paris un pastiche italianisant I'histoire de l'art n'y voyait qu'une tocade puérile. Reine de France pour complaire aux trésoriers et ministres effrayés devant le gouffre abyssal des finances publiques, Marie de Médicis était un porte-monnaie avant même d'être l'épouse du roi. Elle représentait une belle concession à la raison d'État et n'existait que dans l'effacement de sa personnalité. Mais l'histoire se méfie des caricatures. Cette femme, encore si jeune fille, voyait bien d'autres attraits à cette alliance dynastique. Elle aimait son royal époux et malgré l'étrangeté d'une passion qu'un siècle ne pouvait comprendre, elle pardonna toujours les infidélités notoires et les scandaleuses rancœurs d'un homme pour qui elle avait quitté les splendides paysages de Toscane.

Dans cet élan mythifié que tissa l'historiographie traditionnelle, l'ouvrage que consacre au palais du Luxembourg Sara Galletti vient poser les bases de perspectives nouvelles. II permet même de se demander si le palais du Luxembourg ne fut pas l'ultime hommage d'une amante envers l'homme qui lui offrit les honneurs d'une couronne bordée de lys.

En effet, alors que le bon roi Henri tombait sous les coups répétés d'un illuminé, le lendemain même de son couronnement et tout juste dix années après son fastueux mariage, Marie de Médicis se lançait dans I'aventure constructive la plus étonnante du début $d u x \|^{e}$ siècle. Cette aventure, on le sait, fut marquée de nombreux soubresauts. Les " guerres de la mère et du fils " (la reine Marie de Médicis et le jeune Louis XIII) entre 1617 et 1619, I'exil forcé d'une reine qui ne comprenait rien à la politique, puis le retour en grâce et l'accès au Conseil, forment une suite inin-
History of Art, 1953. (rééd. et traduction française, Paris, Macula, 1983).

4. Voir à ce sujet, l'ouvrage commémoratif : Bernard Morice, Le Palais du Luxembourg et ses métamorphoses, ouvrage publié à l'occasion du centenaire du Sénat républicain, s.l., s.n., [1975]. 
terrompue d'allers-retours entre un palais en chantier et des terres éloignées. Tout fut consommé avec la journée des Dupes (10 novembre 1630) qui scella la fin du pouvoir politique de la reine mère au profit d'un tandem improbable composé du roi et de Richelieu. Mais entre 1610 et 1630, que d'activités s'étaient déversées sur le chantier du Luxembourg. Marie, on le sait, fut contrainte à la fuite. Elle quitta le royaume de France dans un périple infini qui la mena dans toutes les cours européennes avant de mourir dans le plus grand dénuement à Cologne chez Rubens, en juillet $1642^{5}$. Elle laissait pourtant une œuvre qui, notamment au travers la grande commande passée à l'atelier Rubens pour la décoration de la galerie, allait devenir, avec le recul, l'expression d'une véritable pensée politique ${ }^{6}$.

Le livre de Sara Galletti s'ouvre par une petite description nostalgique et sensible. Il y est question d'un palais que I'on ne voit plus, qui n'est pratiquement jamais ouvert au public, qui n'existe que par un jardin (si parisien) et une façade (du XIX siècle). C'est donc I'histoire de cette absence qui fabrique l'argument de cette archéologie monumentale.

Autant le dire tout de suite, l'écriture de ce texte est remarquable. La sensibilité qui s'empare des premières pages se retrouve tout au long de l'ouvrage. Mais cette sensibilité de la plume n'est pas un masque à l'analyse, à l'érudition, à la quête obstinée de connaissance. Bien au contraire, un style souple et plaisant, une

5. Helga Hübner, Eva Regtmeier, « Maria de' Medici, eine Fremde, Florenz - Paris Brüssel - London, Köln », Dirk Hoeges (dir.), Dialoghi/Dialogues : Literatur und Kultur Italiens und Frankreichs, Band 14, Francfort, Peter Lang, 2010.

6. Béatrice de Andia et Marie-Noëlle Baudouin-Matuszek (dir.), Marie de Médicis et le palais du Luxembourg, DAAVP, 2000, qui publièrent des documents de première importance sur l'histoire de cette écriture aérée et rigoureuse, une somme de formules saisissantes d'intelligence, un vocabulaire architectural parfaitement maîtrisé transforment la lecture en plaisir savant. On saluera au passage le magnifique travail du traducteur, Julien Noblet, capable d'avoir, à ce point, saisi les inflexions d'une plume.

Mais venons-en au fond, à l'étude, au pari problématique. L'histoire s'ouvrirait donc dans la volonté qu'eut Marie de Médicis d'avoir « une maison pour me loger », rien de plus. Or, dès les premières interventions (1611-1616), Marie se posa non simplement en reine mère, mais en régente. Elle fut celle qui portait I'héritage de la couronne de France et désirait offrir à Paris un monument suprême de l'excellence monarchique. À la fois palais suburbain et palais inscrit dans la ville, le Luxembourg combine, dans son échelle, toutes les contradictions qui avaient opposé le Louvre aux Tuileries, ou Fontainebleau au val de Loire. Le minutieux travail de l'auteur sur la lente captation foncière menée au Luxembourg par les maîtres d'œuvre de Marie raconte parfaitement ce modelage de l'univers urbain au profit d'un Projet. Très vite apparaît ce que Sara Galletti qualifie de "stratégie d'image ». Loin de la sempiternelle référence plagiaire à Pitti et aux relevés exécutés par Salomon de Brosse à Florence, naît l'idée d'une confusion théorique assez proche des errements sémantiques des premiers architectes de l'âge classique. Les campagnes de

construction, venus utilement compléter I'ouvrage majeur de Rosalys Coope (voir supra). travaux dirigées par de Brosse sur la volumétrie d'ensemble (1621-1623), sur l'appartement de la Reine (1621-1625), voient confluer sur ce programme les esprits les plus brillants mais aussi parfois, les plus opposés du temps. Richelieu bien sûr dont le goût très sûr ne peut s'embarrasser d'approximations, mais aussi Claude Maugis, Peiresc, Rubens, Marin de la Vallée, Tommaso Francini, etc., plus tard, Jacques Lemercier, Jean Thiriot, Pierre Scellier, "passèrent » sur ce chantier. On ne sait rien des propos qu'ils échangèrent. Mais tous contribuèrent à penser cette œuvre dans sa complexité, et peut-être même à y introduire davantage encore de complexité. Le pari fut gagnant et I'on sait comment l'architecture française sût puiser à cette source intarissable. L'on pourrait presque reprocher à l'auteur d'avoir quelque peu négligé cette part "communautaire " de I'histoire du Luxembourg. On ne comprend pas bien pourquoi il fallut se détourner de Métezeau', ni même ce qu'apportèrent d'autres architectes parisiens importants comme Le Muet (qui fit la maquette du palais en novembre 1614). On sait par ailleurs que les architectes royaux formaient une équipe complexe dans laquelle il est bien difficile de percevoir des personnalités indépendantes ${ }^{8}$, et il aurait été intéressant de présenter cette logique (encore bien française) du travail en commun. Alors que la signature personnelle s'élevait peu à peu au rang de principe, le Luxembourg offrait
7. Sur Métezeau, voir Emmanuelle Loizeau, Louis et Clément Métezeau, architectes du roi, thèse de doctorat, Paris 4-Sorbonne, 2009.

8. Voir Hilary Ballon, The Paris of Henry IV: Architecture and Urbanism, Cambridge (MA), MIT Press, 1994. 
le privilège d'être le grand chantier vers lequel tous les yeux se tournaient et tous les discours se portaient. Se comprendraient encore mieux alors les beaux développements de Sara Galletti sur l'image du couple royal au travers d'appartements symétriques, sur le principe des logis double (hiver/été, privé/apparat, etc.) qui constituent une hypothèse d'interprétation séduisante et apparemment juste. Avec beaucoup de retenue, l'auteur reste prudente sur ces propositions de lecture. Mais toute la suite de l'ouvrage renforce certaines hypothèses, par ponctuations successives. Mais nous laissons aux lecteurs le plaisir de ces découvertes. En choisissant de présenter une histoire comparative du Luxembourg face à l'architecture et aux programmes de son temps (en France comme en Italie) uniquement après cette tentative d'interprétation, l'auteur nous permet de mieux comprendre sa démarche d'analyse. En revanche, il aurait été sans doute plus intéressant de relier le projet ambitieux du Luxembourg aux grands programmes des Valois plutôt qu'aux références architecturales de la Première Renaissance française pour lesquelles les rapprochements semblent parfois un peu artificiels.

Reprenant une à une les sources d'archives et restituant, le plus fidèlement possible, les partis pris qui s'établirent tout au long de ce vaste chantier ${ }^{9}$, la complexité apparente s'éclaire ${ }^{10}$. Qu'importe alors de savoir si le Luxembourg est un palais (italien) ou un château (français).

9. Il faut reconnaître que l'on bénéficie d'un nombre de relevés et de gravures impressionnant : depuis Marot (1645), Silvestre, Desgodets, quelques anonymes entre 1715 et 1747, en passant par Blondel dans son Architecture françoise de 1752-1756, jusqu'à Gisors lors des travaux sous Louis-Philippe (publication 1847), tout le Luxembourg se croque au fil des ans.
Ce qui importe en revanche, c'est de bien comprendre comment les pavillons d'angle, le noyau central du corps de logis avec l'accès au grand escalier et à la chapelle, le pavillon d'entrée isolé, etc. constituent des réponses opportunes et durablement appréciées. Les élévations elles-mêmes ne sont plus perçues comme la simple interprétation par Salomon de Brosse du modèle de Bartolomeo Ammannati, mais bien comme cette " peau » sur laquelle les bossages, les refends, les ordres d'architecture, se soumettent à une combinatoire qui fabrique une architectonique moderne. Résumer ce livre en quelques paragraphes n'est pas possible, tant il offre d'informations historiques et de pistes de réflexion. Mais il est un point qui s'offre comme une évidence : il s'agit là d'un grand livre. À bien des égards cette monographie, si importante pour l'histoire de l'architecture française du xviie siècle, est aussi un grand livre d'architecture. Ce passage "du territoire à l'édifice », de l'idée au projet et de la formalisation à l'édification, manifeste des qualités d'analyse qui devraient être celles de tout architecte historien. On notera la qualité des pièces graphiques (plans, etc.) dessinées et restituées par l'auteur, qui apporte cette exactitude manquant souvent dans les livres d'histoire de l'architecture. Ellemême architecte, Sara Galletti, manipule des outils, des références historiques, des sources archivistiques et des concepts qui font de cet ouvrage une référence. La

10. On regrettera tout de même que l'auteur n'ait pas davantage utilisé les relevés (transformations) de l'entourage de Soufflot, présentés naguère en partie par Rosalys Coope (op. cit.) et qui présentaient, en filigrane, une interrogation archéologique sur cette construction. très grande qualité des archives présentées et transcrites (correspondances diplomatiques de Marie de Médicis, comptes des bâtiments du roi, nombreux marchés de Marin de la Vallée, de Thiriot, etc.) qui couvrent une période allant de 1612 à 1630, complètent d'ailleurs parfaitement cette étude. Plus que de simples pièces justificatives, elles cellesci témoignent de la complexité d'une œuvre finalement pensée dans sa globalité, mais établie dans l'espérance d'une excellence toujours remise en cause.

Avec cet ouvrage, le palais du Luxembourg se comprend bien comme œuvre mûre et intelligemment menée, et non comme le fruit d'une greffe italienne en val de Seine. Ce fut peut-être cela qui enthousiasma Le Bernin lors de son séjour à Paris en 1664 : le Luxembourg n'était pas le pastiche appauvri de ce qu'il voyait en Italie, mais bien une Architecture.

THIERRY VERDIER

Architecte, docteur en histoire de l'art, agrégé, professeur d'histoire moderne, université Paul-Valéry Montpellier III, président de l'Énsa Toulouse. 